\title{
A Novel Method for the Correlation of Histological Slides and MRI Imaging of the Prostate using 3D Vertex Models
}

\section{Tom Bisson}

University of Applied Sciences

Iris Piwonski

Charite Universitatsmedizin Berlin

\section{Sebastian Lohmann}

Hochschule fur Technik und Wirtschaft Berlin

Norman Zerbe

Charite Universitatsmedizin Berlin

Andreas Maxeiner

Charite Universitatsmedizin Berlin

David Horst

Charite Universitatsmedizin Berlin

Peter Hufnagl

Charite Universitatsmedizin Berlin

\section{Tobias Penzkofer}

Charite Universitatsmedizin Berlin

Sefer Elezkurtaj ( $\square$ sefer.elezkurtaj@charite.de )

Research

Keywords: Histology-MRI Correlation, Prostate Carcinoma, 3D Model

Posted Date: March 13th, 2020

DOI: https://doi.org/10.21203/rs.3.rs-17082/v1

License: (c) (1) This work is licensed under a Creative Commons Attribution 4.0 International License.

Read Full License 


\section{RESEARCH}

2

A Novel Method for the Correlation of 5

Histological Slides and MRI Imaging of the

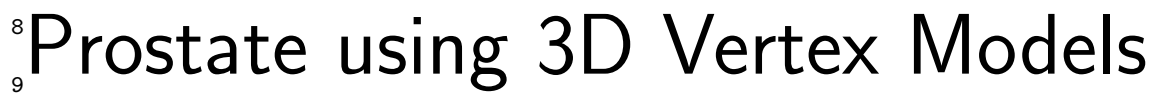

${ }^{10}$ Tom Bisson ${ }^{1,2^{*}}$, Iris Piwonski ${ }^{2}$, Sebastian Lohmann ${ }^{1}$, Norman Zerbe ${ }^{2}$, Andreas Maxeiner $^{3}$, David Horst ${ }^{2},{ }^{10}$ ${ }^{11}$ Peter Hufnag ${ }^{1,2}$, Tobias Penzkofer ${ }^{4,5}$ and Sefer Elezkurtaj ${ }^{2}$ 12

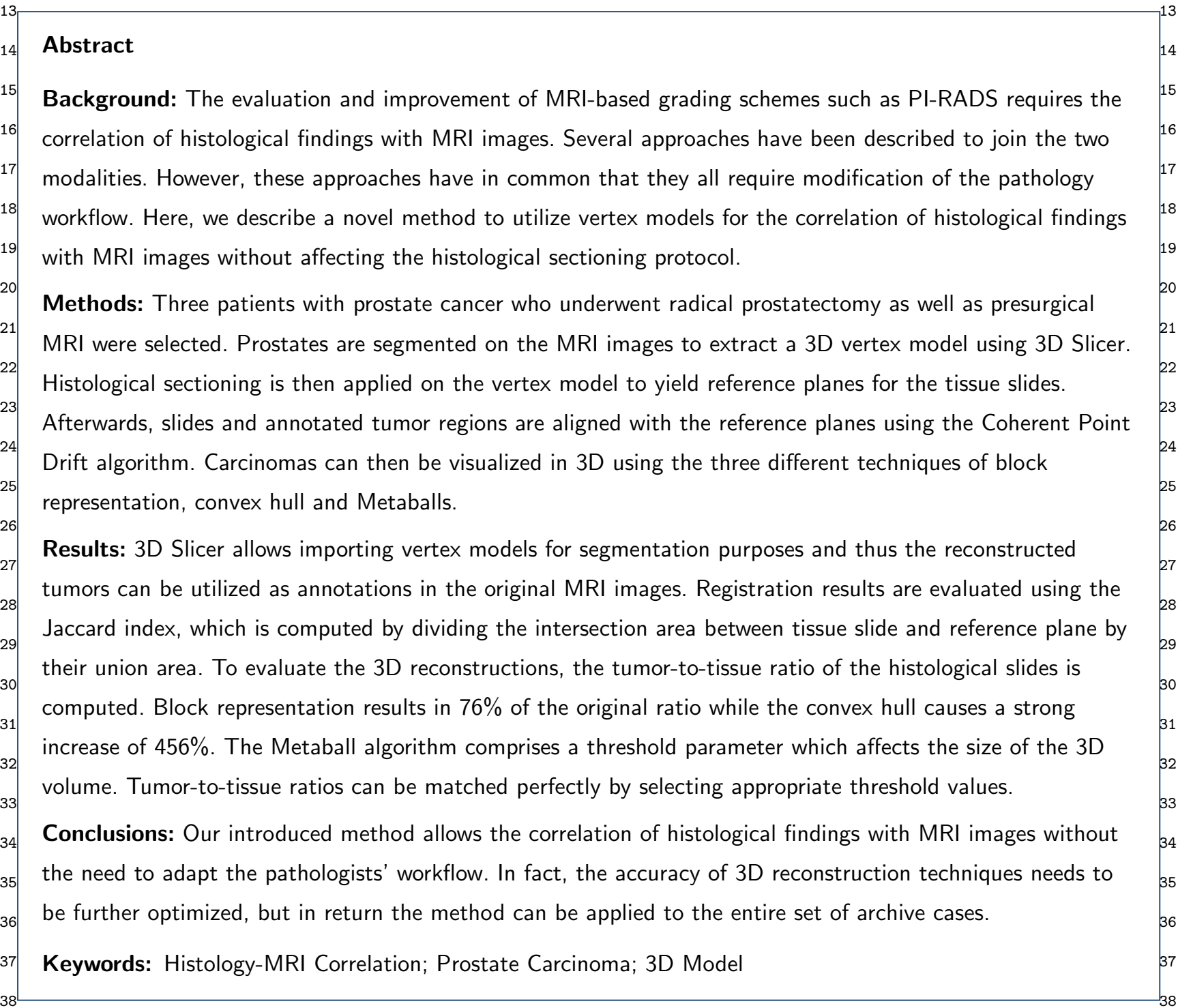




\section{${ }^{1}$ Background}

${ }^{2}$ Core needle biopsies are the gold standard for the di-

${ }^{3}$ agnosis of prostate cancer (PCa). Biopsies are taken ${ }^{4}$ from a set of standard positions which can be ex${ }^{5}$ tended to further biopsies related to lesions visible ${ }^{6}$ in MRI images. Several approaches exist to describe ${ }^{7}$ the visible lesions and estimate their malignancy. Us${ }^{8}$ ing the multi-parametric prostate MRI (mpMRI), the ${ }^{9}$ Prostate Imaging and Reporting Data System (PI${ }^{10}$ RADS) can be utilized to classify morphological as ${ }^{11}$ well as functional features of the prostate in a non${ }^{12}$ invasive manner $[1,2]$. In order to evaluate PI-RADS ${ }^{13}$ and alternative grading schemes, a method is required ${ }^{14}$ that allows for the transfer of histological findings to ${ }^{15}$ the corresponding regions in the MRI images. Since ${ }^{16}$ the histological slides cannot be well aligned with the ${ }^{17} \mathrm{MRI}$ images, their correlation requires different prepa${ }^{18}$ ration techniques [3]. Several approaches for the regis${ }^{19}$ tration of histological slides to MRI images have been ${ }^{20}$ described, all of which entail special effort and changes ${ }^{21}$ in the processing routine. Furthermore, difficulties may ${ }^{22}$ arise when applying these techniques to archival cases ${ }^{23}$ which have been prepared according to the typical rou${ }^{24}$ tine practice. Here, we describe a novel method for the ${ }^{25}$ correlation of histological slides with MRI images of ${ }^{26}$ the prostate using $3 \mathrm{D}$ vertex models. 27 ${ }_{28}$ Related Work

${ }_{29}$ Several papers have been published about methods soto correlate histological slides of the prostate with ${ }_{31} \mathrm{MRI}$ data. Artificial reference points can be created ${ }_{32}$ by punctuating the prostate with thin needles which з3allows an inter-slide-registration to facilitate three-

$34^{*}$ Correspondence: tom.bisson@charite.de

${ }^{1}$ Center for Biomedical Image and Information Processing, University of

Applied Sciences (HTW) Berlin, Wilhelminenhofstr. 75 A, 12459 Berlin, ${ }^{36}$ Germany

$37^{2}$ Charité - Universitätsmedizin Berlin, corporate member of Freie Universität Berlin, Humboldt-Universität zu Berlin, and Berlin Institute of Health, Institute of Pathology, Charitéplatz 1, 10117 Berlin, Germany

${ }^{39}$ Full list of author information is available at the end of the article dimensional alignment [4]. Other approaches focus on ${ }^{1}$ performing the sectioning according to the recording ${ }^{2}$ angle of the MRI in order to directly register histo- $^{3}$ logical slides to the corresponding image $[5,6]$. Most ${ }^{4}$ pathology institutes do not use whole mounts to avoid ${ }^{5}$ the various obstacles to histological processing and ${ }^{6}$ compatibility with archiving issues. For this reason, ${ }^{7}$ methods have been developed to register further sub- ${ }^{8}$ divided tissue slices. This can be done by photograph- ${ }^{9}$ ing the tissue slice before further subdivision. The im- ${ }^{10}$ age can then be used to reassemble the partial slices ${ }^{11}$ into a whole [7]. In order to register the joined tis- ${ }^{12}$ sue slices onto the corresponding MRI images, both ${ }^{13}$ can be converted into three-step grayscale images [8]. ${ }^{14}$ Finally, a device was introduced that allows the ac- ${ }^{15}$ quisition of MRI images of the ex vivo specimen with ${ }^{16}$ correspondingly sliced sections of the prostate, thus ${ }^{17}$ providing optimal data for correlation [9]. In addition ${ }^{18}$ to the methods for image registration, several publi- ${ }^{19}$ cations describe patient-specific mounts derived from ${ }^{20}$ MRI data that allow high-precision sectioning of the ${ }^{21}$ prostate $[10,11,12,13]$.

\section{Methods}

24

Patients and image acquisition

25

Three different patients (age ranging from 59 to $75^{26}$ years, Gleason $3+4=7$ (ISUP 2), UICC stadium ${ }^{27}$ II and III (pT2c - pT3b), one patient with tumor ${ }^{28}$ in resection-boundaries (R1), all patients with per- ${ }^{29}$ ineural invasion $(\mathrm{pN} 1)$ but without regional $\mathrm{ymph}^{30}$ node metastasis ( $\mathrm{pN} 0$ ), lymphovascular or vascular ${ }^{31}$ invasion (L0V0)) who underwent radical prostatec- ${ }^{32}$ tomy have been chosen for the development of the ${ }^{33}$ method. All slides were digitalized using either the ${ }^{34}$ 3DHistech Pannoramic SCAN II (P150) or the Hama- ${ }^{35}$ matsu Nanozoomer and tumor regions were annotated ${ }^{36}$ using Cognition Master Professional Suite (v4.0) by ${ }^{37}$ VMscope. MRI was acquired using a Siemens Health-38 ineers Skyra 3T MRI scanner and the prostate $\operatorname{was}^{39}$ 
${ }^{1}$ segmented with 3D Slicer v4.0 ([14]) and converted ${ }^{2}$ into a $3 \mathrm{D}$ vertex model.

3

\section{${ }^{4}$ Correlation of histological slides with MRI}

${ }^{5}$ Histological sectioning of the prostate is transferred 6to the MRI-derived 3D vertex model, followed by ap${ }^{7}$ plying point set registration of the tissue slides to the 8approximated slices in the 3D model. To apply the ${ }^{9}$ sectioning to the $3 \mathrm{D}$ vertex model of the prostate, the ${ }^{10}$ open source 3D graphics suite Blender v2.79b is used. ${ }^{11}$ The supplied Boolean modifiers can be used to cre12ate sectional planes through $3 \mathrm{D}$ vertex models, allow13ing the sectioning of the ex vivo specimen to be fully ${ }^{14}$ transferred. Figure 1 illustrates this process by plac15ing a section through a vertex model and then further ${ }^{16}$ dividing it into quarters.

17 In order to transfer the sectioning process to the 3D ${ }^{18}$ model with the necessary fidelity, the dissection pro${ }^{19}$ tocol needs to be considered. Therefore, we created a ${ }^{20}$ JSON scheme that allows to digitally represent the in${ }^{21}$ dividual sub-slices along with their position and slide ${ }^{22} \mathrm{ID}$. In addition, the fragmentation of the preparation ${ }^{23}$ according to the NeuroSAFE technique for intraopera${ }^{24}$ tive consultation ([15]) must be taken into account. To ${ }^{25}$ this end, we again used Blender's boolean modifier to ${ }^{26}$ remove the approximated NeuroSAFE segments from ${ }^{27}$ the specimen, as displayed in figure 2 .

28 Applying the sectioning to the 3D models yields a set ${ }^{29}$ of approximated slices in $3 \mathrm{D}$ space, serving as a refer${ }^{30}$ ence model for the registration of histological slides. ${ }^{31}$ Since the vertex model only consists of polygonal sur${ }^{32}$ faces representing the prostate capsule, the reference ${ }^{33}$ slides are comprised solely of vertices and connecting ${ }^{34}$ edges. Therefore, the registration is limited to area ${ }^{35}$ and shape-based algorithms, such as coherent point ${ }^{36} \mathrm{drift}$ (CPD) [16]. Since CPD requires pre-registration, ${ }^{37}$ all approximated sections are transferred to $2 \mathrm{D}$ space ${ }^{38}$ and then used for alignment and isotropic scaling of ${ }^{39}$ the histological sections. While translation and scal- ing are easy to compute, the determination of the best ${ }^{1}$ initial rotation is not trivial and therefore a series of ${ }^{2}$ incrementing rotation angles are used. The registra- ${ }^{3}$ tion results are then compared using the Jaccard in- ${ }^{4}$ $\operatorname{dex}([17])$ and best parameters are used for the final ${ }^{5}$ registration. Since we have found that an increasing ${ }^{6}$ number of polygon vertices leads to better registration ${ }^{7}$ results, we have implemented an algorithm to extrap- ${ }^{8}$ olate polygons while maintaining their shape. Starting ${ }^{9}$ with the starting polygon, the longest edge is located ${ }^{10}$ and a new vertex is inserted at half the edges' length. ${ }^{11}$ This process is repeated until the target number of ${ }^{12}$ vertices has been reached for both the prostate slide ${ }^{13}$ outlines and the corresponding MRI reference poly- ${ }^{14}$ gons. Subsequently, all histological slides including tu- ${ }^{15}$ mor regions are registered on their reference polygons, ${ }^{16}$ as shown in Figure 3.

\section{7} 18

After registration, all tumor regions are retransferred ${ }^{21}$ to their associated positions in 3D space resulting in $\mathrm{a}^{22}$ set of polygons which represent the tumor outlines at ${ }^{23}$ the given locations. Based on these polygons the $3 \mathrm{D}^{24}$ tumor volume can be rendered from the annotations ${ }^{25}$ using different approaches. We implemented three ren- ${ }^{26}$ dering techniques and evaluated them by comparing ${ }^{27}$ the resulting tumor-to-tissue ratio with the ratio de-28 rived from the histological slides. In the first approach, ${ }^{29}$ all polygons are thickened according to the approx- ${ }^{30}$ imated tissue block size. Second, the convex hull of ${ }^{31}$ all polygons is computed. The third visualization aims ${ }^{32}$ at an interpolation of the tumor regions by utilizing ${ }^{33}$ Metaballs ([18]), which belong to the family of im- ${ }^{34}$ plicit surfaces. Only the latter approach can be param- ${ }^{35}$ eterised in the form of a threshold value with which the ${ }^{36}$ merging behaviour of the Metaballs can be specified. ${ }^{37}$ A higher threshold penalizes the influence on other ${ }^{38}$ Metaballs and leads to smaller Metaballs in general. ${ }^{39}$ 


\section{${ }^{1}$ Results}

${ }^{2}$ As deformations occur during the dissection and 3 preparation of the prostate, the $3 \mathrm{D}$ models derived from the MRI differ from the ex vivo specimens and 5 an additional change in shape is caused by the Neu6 roSAFE technique. To quantify the differences, we 7 measured the ex vivo specimens of all three patients 8 and compared them to the dimensions of the 3D mod9 els derived from MRI images. The results are given in 10 table 1 . 11 12 The alterations of size do not occur constantly along ${ }_{13}$ a particular axis or in a specific patient. This might be ${ }_{14}$ related to the NeuroSAFE technique, which involves ${ }_{15}$ removal of a posterolateral portion of the prostate fol${ }_{16}$ lowing radical prostatectomy. However, the apicobasal ${ }_{17}$ axis is usually not affected by this technique and yet ${ }_{18}$ the deviation from the 3D model varies between $1.06 \%$ 19 and $1.20 \%$.

20 Polygon registration has been performed using CPD 21and the results were evaluated with the Jaccard in22dex. All polygons have been extrapoliert to 500 points 23and 8 initial rotations each differing by an angle of $45^{\circ}$ 24are used. The mean results of the three cases (Addi25tional File 1: Registration Results) were 0.87, 0.86 and 260.91 with a standard deviation of $0.05,0.07$ and 0.04 , 27respectively. Figure 4 shows representative images for 28the three volume rendering techniques.

29 All three techniques are evaluated by comparing the ${ }^{30}$ resulting tumor-tissue ratio with the one in the his-

${ }^{31}$ tological sections. The block representation results in ${ }^{32}$ a smaller ratio, making up only $76 \%$ of the refer${ }^{33}$ ence values while the convex hull causes a strong in${ }^{34}$ crease of $456 \%$. For the evaluation of the metaballs, the ${ }^{35}$ algorithm's threshold parameter must be considered. ${ }^{36}$ When each case is parametrized independently, the ref${ }^{37}$ erence values can be matched explicitly by choosing ${ }^{38}$ individual thresholds of $1.824,1.936$ and 0.987 . When ${ }^{39}$ the $3 \mathrm{D}$ reconstruction is complete, the vertex models can be reimported into $3 \mathrm{D}$ slicer to provide segmenta- ${ }^{1}$ tion of the MRI images. 2

\section{Discussion}

To fit on standard size slides, tissue sections must be ${ }^{5}$ further subdivided, which can lead to additional tissue ${ }^{6}$ deformations and defects. Whole mount sectioning can $^{7}$ be used to solve these problems $([19,3])$, which further ${ }^{8}$ complicates histological processing and archiving and ${ }^{9}$ is therefore only used by a small part of the urological ${ }^{10}$ pathologists [20]. Thus, we advocate a digital solution ${ }^{11}$ to realign the sub-slices prior to registration with the $\mathrm{e}^{12}$ reference model.

Metaballs give the most realistic impression of the $\mathrm{e}^{14}$ tumors when comparing the three visualization meth- ${ }^{15}$ ods presented. The tumor-to-tissue ratio of the his- ${ }^{16}$ tological slides can be matched perfectly by adjusting ${ }^{17}$ the threshold parameter. However, an individually dif-18 ferent value has to be determined for every case. Ad-19 ditionally, the detailed information about the tumor ${ }^{20}$ contours may be impaired. The convex hull is inappro- ${ }^{21}$ priate for the visualization of $\mathrm{PCa}$ as it causes loss of ${ }^{22}$ information about the spatial distribution of multiple ${ }^{23}$ tumor foci. Using block representation, the tumor-to- ${ }^{24}$ tissue ratio is below the reference values. This might $b^{25}$ related to both registration errors as well as the miss- ${ }^{26}$ ing interpolation between the slices. While the visual-27 ization appears to be very schematic it is well-suited ${ }^{28}$ to describe the distribution and outline of the PCa. ${ }^{29}$ However, it does not provide a sound base for interpo- ${ }^{30}$ lation, since the underlying information does not al- ${ }^{31}$ low a spatial assignment between the different tumor ${ }^{32}$ regions.

At the current stage, we did not consider different ${ }^{34}$ Gleason grades or growth patterns. All tumor lesions ${ }^{35}$ were treated equally, and no distinctions are made. ${ }^{36}$ This could be easily implemented in the block-wise ${ }^{37}$ visualization where every block is handled as an indi- ${ }^{38}$ vidual object and can be labelled independently. To ${ }^{39}$ 
${ }^{1}$ implement this with Metaballs, each group can be re${ }^{2}$ constructed separately, yielding groups of Metaballs ${ }^{3}$ for each occurring pattern or grade, respectively. 4

\section{${ }_{5}$ Conclusions}

6Radiological-pathological correlation is the corner7stone of any diagnostic paradigm, and highly valid sdatasets are essential for further refinement of diag9nostic tools such as PI-RADS. Our introduced method 10can help to provide a large-scale database for the eval11uation and improvement of PI-RADS by leveraging 12standard pathological diagnostic schemes without ad13ditional processing steps. For this purpose, the MRI14based tumor predictions can be imported into the 3D 15model and a comparison between the predictions and 16the histopathological findings can be made. The accu17racy of our method requires further optimization, but 18in turn, it can be applied to the whole set our archival 19cases.

20

\section{Abbreviations}

${ }^{21}$ MRI: Magnetic Resonance Imaging; mpMRI: multi-parametric MRI 22PI-RADS: Prostate Imaging-Reporting and Data System; PCa: Prostate ${ }_{23}$ Cancer; CPD: Coherent Point Drift.

${ }_{24}$ Ethics approval and consent to participate

MRI data postprocessing was approved by Charité - Universitätsmedizin ${ }^{25}$ Berlin (EA1/271/16).

26

Consent for publication

${ }^{27}$ Not applicable.

${ }^{28}$ Availability of data and materials

29All data is available from the corresponding author upon reasonable request.

\section{Competing interests}

${ }_{31}$ The authors declare that they have no competing interests.

${ }_{32}$ Funding

Not applicable.

33

Author's contributions

${ }^{34}$ TB and SE developed methodology and performed experiments; TB, IP,

${ }^{35} \mathrm{SL}, \mathrm{NZ}, \mathrm{AM}, \mathrm{DH}, \mathrm{PH}, \mathrm{TB}$ and $\mathrm{SE}$ analyzed and interpreted data and wrote

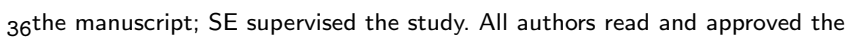
$37^{\text {final manuscript. }}$

${ }_{38}$ Acknowledgements

We acknowledge support from the German Research Foundation (DFG) and 39 the Open Access Publication Fund of Charité - Universitätsmedizin Berlin.
Author details 1

${ }^{1}$ Center for Biomedical Image and Information Processing, University of 2 Applied Sciences (HTW) Berlin, Wilhelminenhofstr. 75 A, 12459 Berlin, 3 Germany. ${ }^{2}$ Charité - Universitätsmedizin Berlin, corporate member of Freie Universität Berlin, Humboldt-Universität zu Berlin, and Berlin Institute of 4 Health, Institute of Pathology, Charitéplatz 1, 10117 Berlin, Germany. 5 ${ }^{3}$ Charité - Universitätsmedizin Berlin, corporate member of Freie 6 Universität Berlin, Humboldt-Universität zu Berlin, and Berlin Institute of Health, Institute of Urology, Charitéplatz 1, 10117 Berlin, Germany.

${ }^{4}$ Charité - Universitätsmedizin Berlin, corporate member of Freie 8 Universität Berlin, Humboldt-Universität zu Berlin, and Berlin Institute of 9 Health, Institute of Radiology, Augustenburger Platz 1, 13353 Berlin,

Germany. ${ }^{5}$ Berlin Institute of Health (BIH), Anna-Louisa-Karsch-Straße 2, ${ }^{10}$ 10178 Berlin, Germany. 11

References 12

1. Weinreb JC, Barentsz JO, Choyke PL, Cornud F, Haider MA, Macura 13 $\mathrm{KJ}$, et al. PI-RADS Prostate Imaging - Reporting and Data System: 14 2015, Version 2. Eur Urol. 2016 Jan;69(1):16-40.

2. Turkbey B, Rosenkrantz AB, Haider MA, Padhani AR, Villeirs G, 15 Macura KJ, et al. Prostate Imaging Reporting and Data System 16 Version 2.1: 2019 Update of Prostate Imaging Reporting and Data 17 System Version 2. Eur Urol. 2019 Sep;76(3):340-351.

3. Fedorov A, Penzkofer T, Hirsch MS, Flood TA, Vangel MG, Masry P, 18 et al. The role of pathology correlation approach in prostate cancer 19 index lesion detection and quantitative analysis with multiparametric 20 MRI. Acad Radiol. 2015 May;22(5):548-555.

4. Bart S, Mozer P, Hemar P, Lenaour G, Comperat E, Renard-Penna R, 21 et al. MRI-histology registration in prostate cancer. In: Proceedings of 22 Surgetica. Sauramps Medical Montpellier France; 2005. p. 361-367. 23

5. Chappelow J, Bloch BN, Rofsky N, Genega E, Lenkinski R, DeWolf W, et al. Elastic registration of multimodal prostate MRI and histology via ${ }^{24}$ multiattribute combined mutual information. Med Phys. 201125 Apr;38(4):2005-2018

Xiao G, Bloch BN, Chappelow J, Genega EM, Rofsky NM, Lenkinski RE, et al. Determining histology-MRI slice correspondences for $\quad 27$ defining MRI-based disease signatures of prostate cancer. Comput 28 Med Imaging Graph. 2011;35(7-8):568-578

7. Orczyk C, Mikheev A, Rosenkrantz AB, Melamed J, Taneja SS, 29 Rusinek H. Imaging of prostate cancer: a platform for 3D 30 co-registration of in-vivo MRI ex-vivo MRI and pathology. Proc SPIE 31 Int Soc Opt Eng. 2012 Feb;8316:83162M.

8. Kalavagunta C, Zhou X, Schmechel SC, Metzger GJ. Registration of 32 in vivo prostate MRI and pseudo-whole mount histology using Local 33 Affine Transformations guided by Internal Structures (LATIS). J Magn34 Reson Imaging. 2015 Apr;41(4):1104-1114.

9. Bourne RM, Bailey C, Johnston EW, Pye $\mathrm{H}$, Heavey S, Whitaker $\mathrm{H}$ et al. Apparatus for Histological Validation of In Vivo and Ex Vivo 36 Magnetic Resonance Imaging of the Human Prostate. Front Oncol. 37 2017;7:47.

10. Shah V, Pohida T, Turkbey B, Mani H, Merino M, Pinto PA, et al. A method for correlating in vivo prostate magnetic resonance imaging $\quad 39$ 
1 and histopathology using individualized magnetic resonance-based 2 molds. Rev Sci Instrum. 2009 Oct;80(10):104301.

31. Turkbey B, Pinto PA, Mani H, Bernardo M, Pang Y, McKinney YL, et al. Prostate cancer: value of multiparametric MR imaging at $3 \mathrm{~T}$ for detection-histopathologic correlation. Radiology. 2010

5 Apr;255(1):89-99.

6 ${ }^{12}$. Trivedi H, Turkbey B, Rastinehad AR, Benjamin CJ, Bernardo M, Pohida T, et al. Use of patient-specific MRI-based prostate mold for validation of multiparametric MRI in localization of prostate cancer

8 Urology. 2012 Jan;79(1):233-239

913. Priester A, Natarajan S, Le JD, Garritano J, Radosavcev B, Grundfest $W$, et al. A system for evaluating magnetic resonance imaging of

10 prostate cancer using patient-specific 3D printed molds. Am J Clin 11 Exp Urol. 2014;2(2):127-135.

12 14. Fedorov A, Beichel R, Kalpathy-Cramer J, Finet J, Fillion-Robin JC, Pujol S, et al. 3D Slicer as an image computing platform for the

13 Quantitative Imaging Network. Magn Reson Imaging. 2012

14 Nov;30(9):1323-1341.

15 15. Schlomm T, Tennstedt P, Huxhold C, Steuber T, Salomon G, Michl U, et al. Neurovascular structure-adjacent frozen-section examination

16 (NeuroSAFE) increases nerve-sparing frequency and reduces positive

17 surgical margins in open and robot-assisted laparoscopic radical

18 prostatectomy: experience after 11,069 consecutive patients. Eur Urol. 2012 Aug;62(2):333-340.

19 16. Myronenko A, Song $X$. Point set registration: coherent point drift.

20 IEEE Trans Pattern Anal Mach Intell. 2010 Dec;32(12):2262-2275.

22 dans quelques régions voisines. Bull Soc Vaudoise Sci Nat. 1901;37:241-272.

2318. Blinn JF. A generalization of algebraic surface drawing. ACM

24 transactions on graphics (TOG). 1982;1(3):235-256.

19. Yamamoto H, Nir D, Vyas L, Chang RT, Popert R, Cahill D, et al. A

25 Workflow to Improve the Alignment of Prostate Imaging with

26 Whole-mount Histopathology. Acad Radiol. 2014

27 Aug;21(8):1009-1019.

20. Samaratunga H, Montironi R, True L, Epstein JI, Griffiths DF,

Humphrey PA, et al. International Society of Urological Pathology

29 (ISUP) Consensus Conference on Handling and Staging of Radical

30 Prostatectomy Specimens. Working group 1: specimen handling. Mod

30

Pathol. 2011 Jan;24(1):6-15

31

32 Figures

33

34

Figure 1 Sectioning of 3D vertex models. Boolean Modifier 6 in Blender for the intersection of a plane with the 3D vertex model of the prostate ( $a \& b$ ), followed by further subdivision into quarters (c).
${ }_{21} 17$. Jaccard P. Distribution de la flore alpine dans le bassin des Dranses et

Figure 2 NeuroSAFE technique on a vertex model. 3D vertex model of the prostate including NeuroSAFE sectional planes (a), and 3D vertex model after removal of the NeuroSAFE segments (b).

Figure 3 Slide registration. Registration of a histological slide (a) to its' 3D approximate with the coherent point drift algorithm. Annotated tumor regions are represented in blue and in the results (b) the slide outline is shown in yellow and the approximate in black.

10

Figure 4 Prostate tumor renderings. Three techniques for rendering tumor volumes in the prostate using (from left to right) block representation, convex hull and metaballs.

Table 1 Dimensions of the ex vivo specimens and the deviation in ${ }^{14}$ the MRI derived 3D models along the horizontal, antero-dorsal 15 and apico-basal axis.

16

\begin{tabular}{c|cc|cc|ccc}
\hline & \multicolumn{2}{|c|}{ Case 1 } & \multicolumn{2}{c|}{ Case 2 } & \multicolumn{3}{c}{ Case 3 } \\
\hline Origin & Ex Vivo & MRI & Ex Vivo & MRI & Ex Vivo & MRI \\
Horizont. & $50 \mathrm{~mm}$ & $1.1 \%$ & $50 \mathrm{~mm}$ & $1.0 \%$ & $53 \mathrm{~mm}$ & $1.1 \%$ & 18 \\
Ant.-Dors. & $41 \mathrm{~mm}$ & $0.9 \%$ & $42 \mathrm{~mm}$ & $1.0 \%$ & $46 \mathrm{~mm}$ & $1.0 \%$ & 19 \\
Ap.-Basal & $30 \mathrm{~mm}$ & $1.2 \%$ & $52 \mathrm{~mm}$ & $1.1 \%$ & $46 \mathrm{~mm}$ & $1.2 \%$ & \multicolumn{3}{c}{20} \\
\hline
\end{tabular}

Tables

Additional Files

Additional file 1: Registration Results

The results of all registrations per case as .xlsx file (MS Excel). 24 


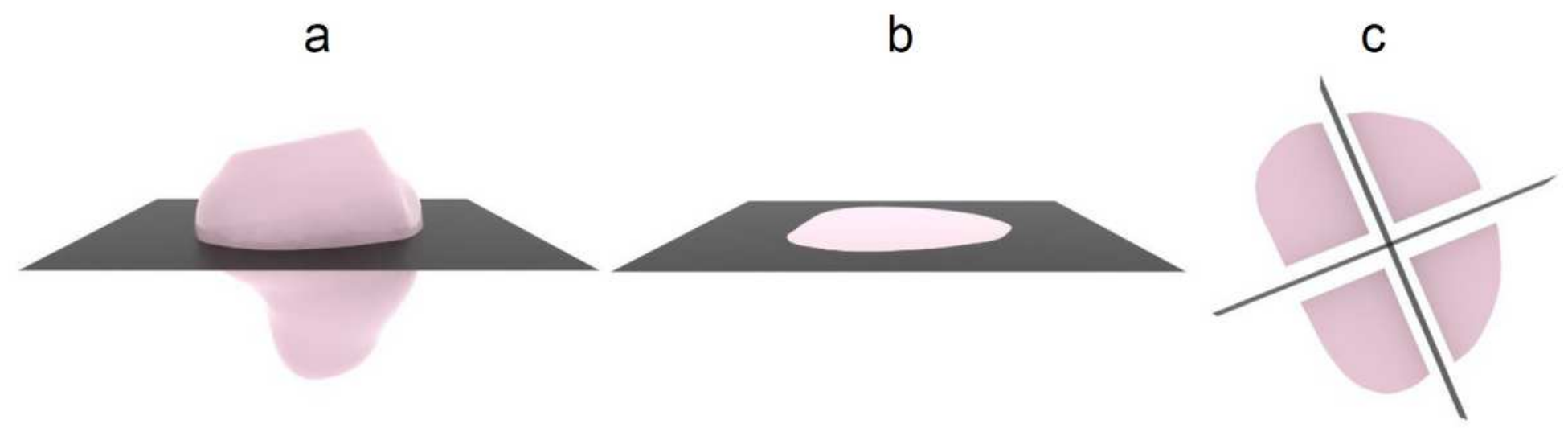

\section{Figure 1}

Sectioning of 3D vertex models. Boolean Modier in Blender for the intersection of a plane with the 3D vertex model of the prostate ( $\mathrm{a} \& \mathrm{~b})$, followed by further subdivision into quarters (c).

a

b
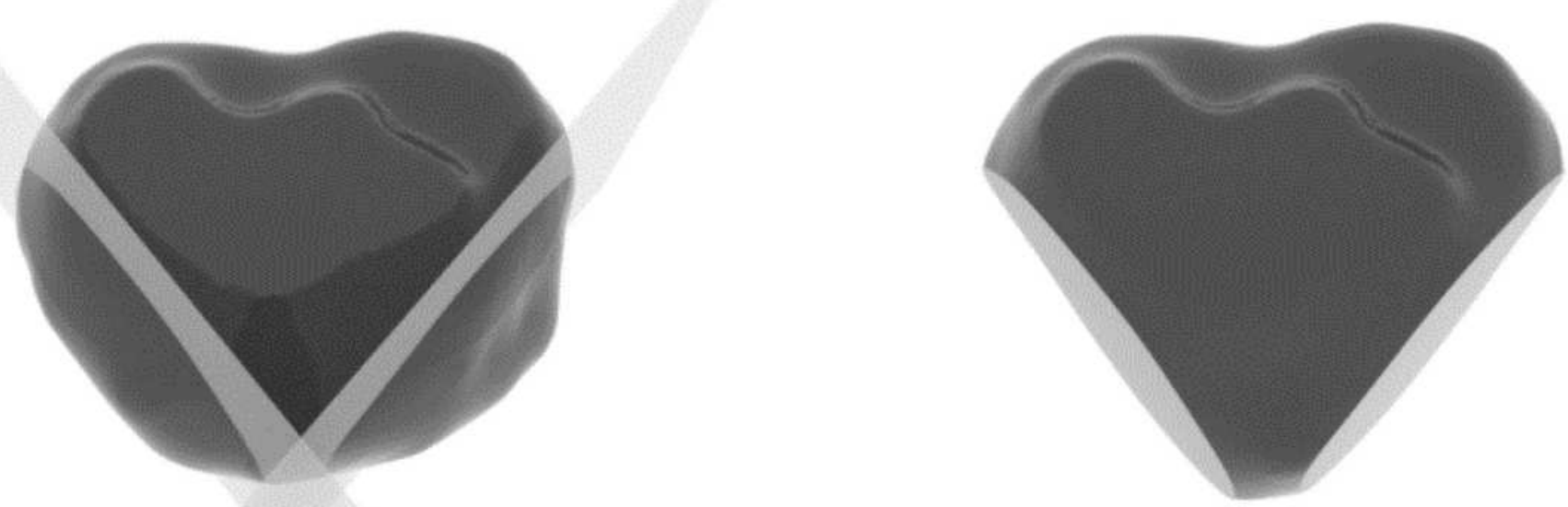

\section{Figure 2}

NeuroSAFE technique on a vertex model. 3D vertex model of the prostate including NeuroSAFE sectional planes (a), and 3D vertex model after removal of the NeuroSAFE segments (b). 
a

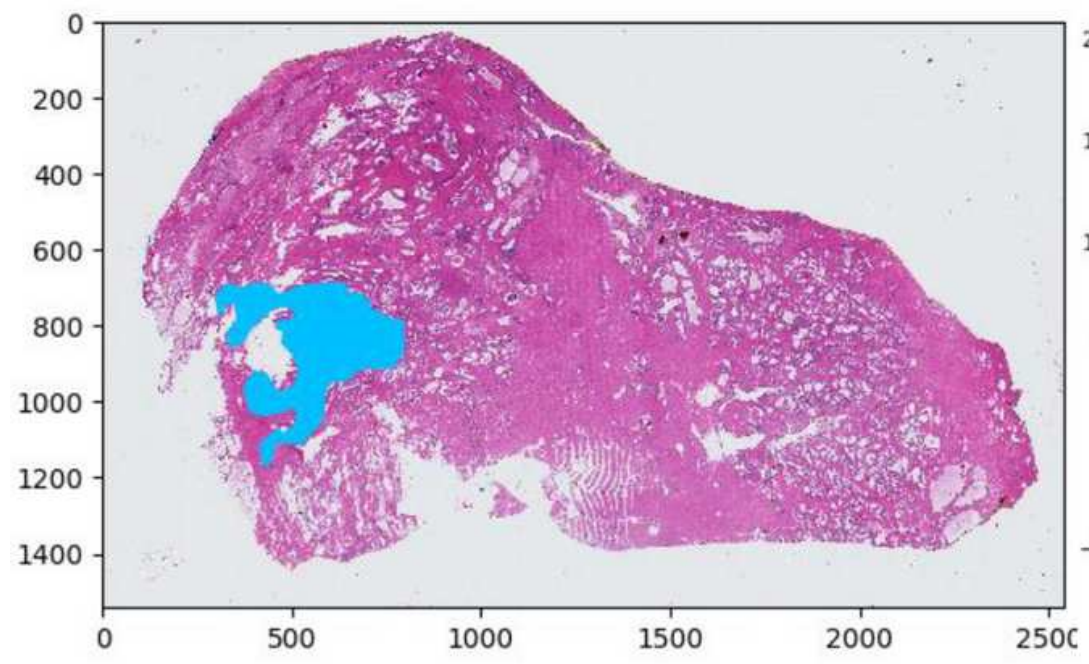

b

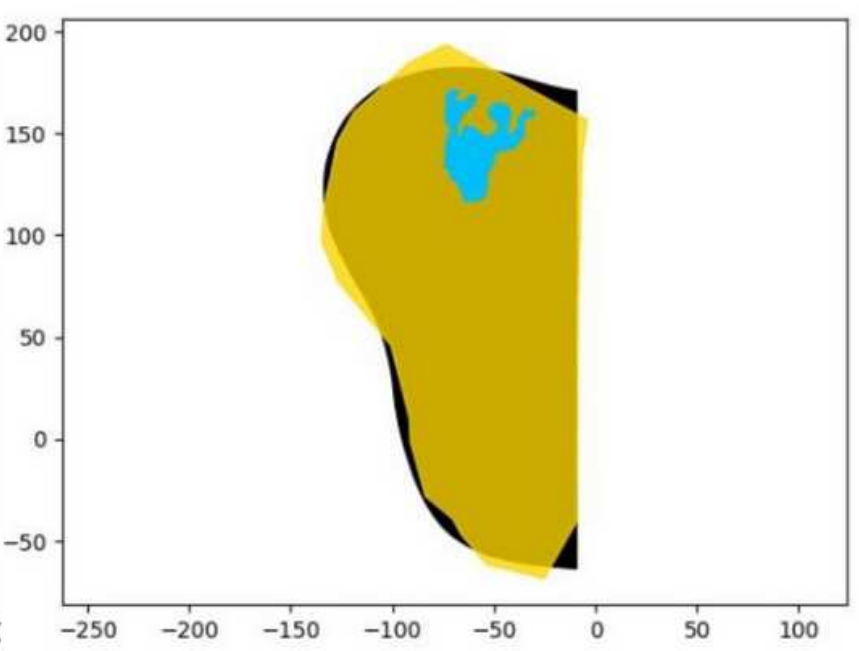

Figure 3

Slide registration. Registration of a histological slide (a) to its' 3D approximate with the coherent point drift algorithm. Annotated tumor regions are represented in blue and in the results $(b)$ the slide outline is shown in yellow and the approximate in black. 
a
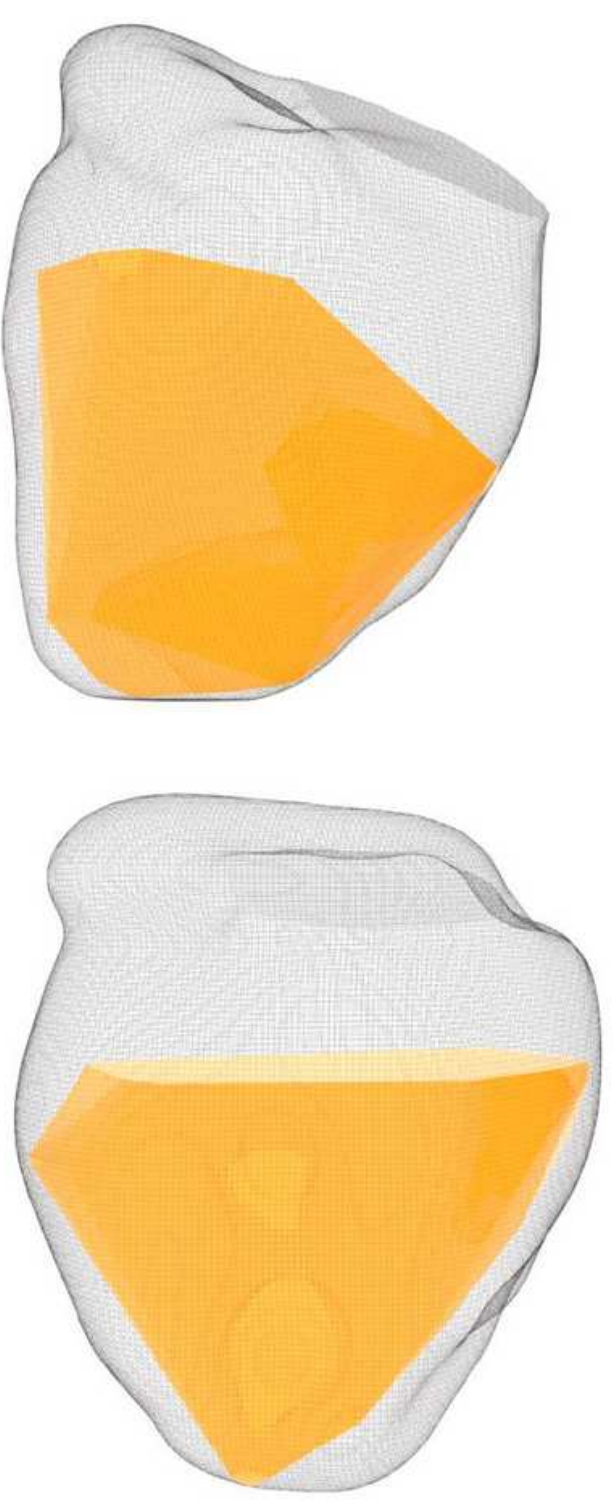

b
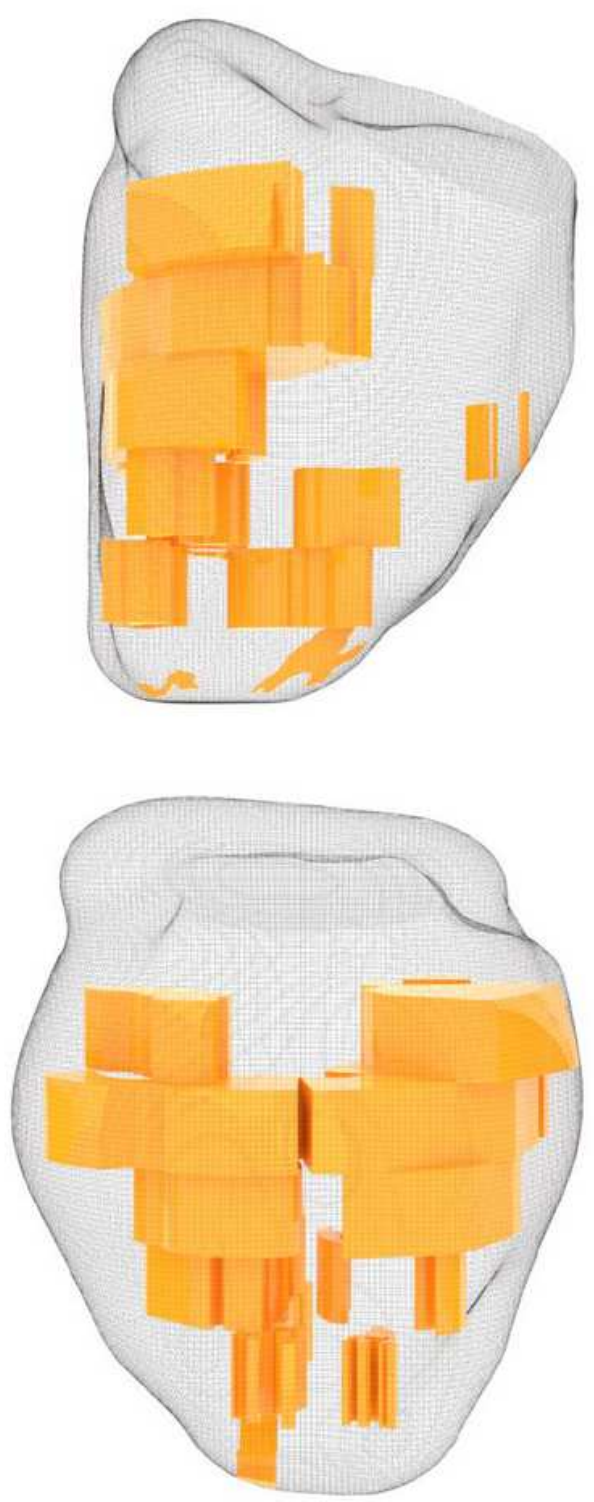

C
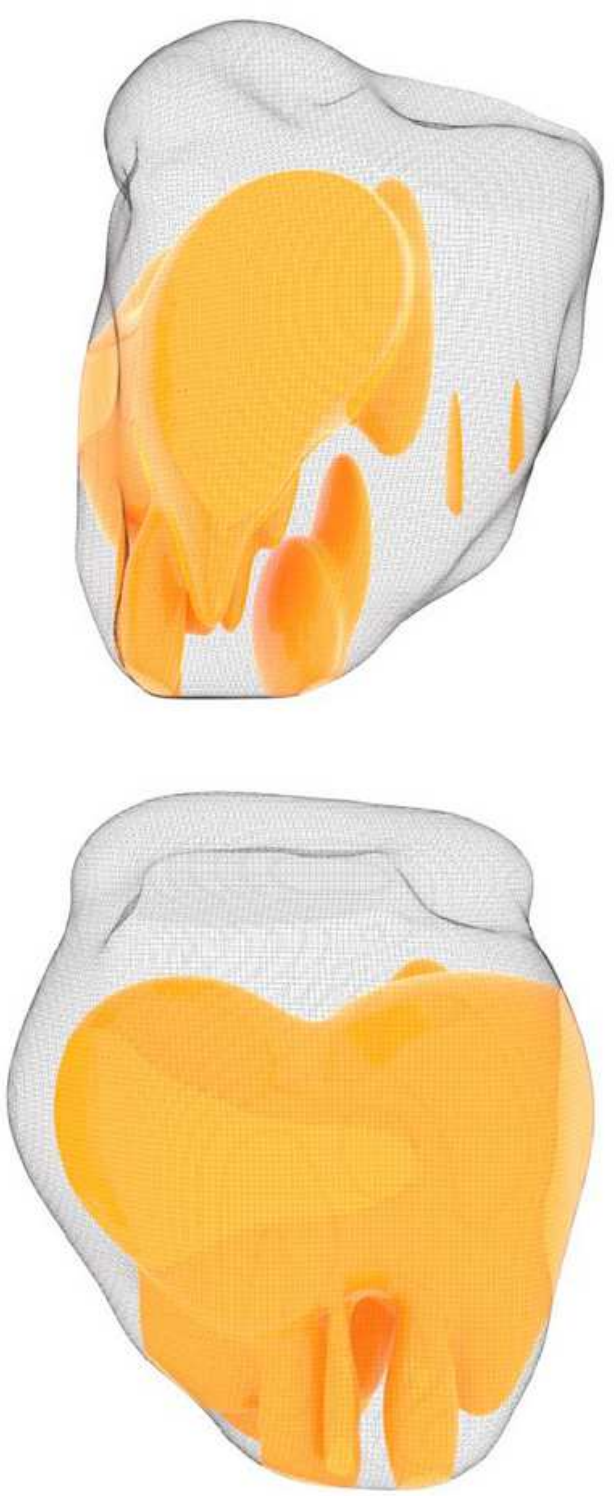

Figure 4

Prostate tumor renderings. Three techniques for rendering tumor volumes in the prostate using (from left to right) block representation, convex hull and metaballs.

\section{Supplementary Files}

This is a list of supplementary files associated with this preprint. Click to download.

- RegistrationResults.xlsx 